\title{
Emotion dysregulation in adults with attention deficit hyperactivity disorder: a meta-analysis
}

\author{
Ashkan Beheshti ${ }^{*}$, Mira-Lynn Chavanon and Hanna Christiansen
}

\begin{abstract}
Background: Emotional symptoms are increasingly considered a core feature of attention deficit/hyperactivity disorder (ADHD). We aimed to quantify the evidence of emotional dysregulation and its respective facets in individuals with adult ADHD compared to healthy controls using meta-analysis.

Methods: Two electronic databases (PubMed, PsycINFO) were reviewed to identify studies. Studies were eligible for inclusion that had reports on any measure of emotion (dys) regulation in adults ( $>18$ years of age) in clinically diagnosed patients with ADHD as well as healthy control participants. We included a total of 13 studies ( $N=2535)$ to assess (1) the standardized mean difference in emotion dysregulation (ED) as a general factor and its specific facets (i.e., emotional lability, negative emotional responses, and emotion recognition) between adults with ADHD and healthy controls; and (2) the association between ADHD symptom severity and ED.

Results: Compared to healthy controls, adults with ADHD revealed significantly higher levels of general ED (Hedges' $g=1.17, p<0.001$; Hedges' $g$ is the adjusted effect size). With regard to intermediate dimensions of ED, emotional lability exhibited the strongest weighted effect (Hedges' $\mathrm{g}=1.20, \mathrm{Cl}[0.57,1.83], p<0.001)$. Furthermore, symptom severity and general ED correlated significantly $(r=0.54, p<0.001)$. Regarding intermediate dimensions of $E D$, negative emotional responses correlated closely with ADHD symptom severity $(r=0.63, p<0.001)$ and emotional lability $(r=0.52, p<0.001)$.

Conclusions: Our findings support ED symptoms as a core feature of ADHD's psychopathology. With respect to dimensions of ED, emotional lability, and negative emotional responses play a more definitive role in the psychopathology of adults with ADHD. Due to insufficient statistical reports in the included studies, we could not perform meta-regressions to control the role of moderator variables.
\end{abstract}

Keywords: ADHD, Emotion dysregulation, adults, Meta-analysis

\section{Background}

Attention Deficit Hyperactivity Disorder (ADHD) is characterized by its core symptoms inattention, impulsivity, and hyperactivity [1]. The past decade of research revealed that ADHD persists into adulthood [2-4]. Apart from the core symptoms, emotion regulation contributes independently to functional impairments in patients with ADHD [5-7]. In this regard, several studies reported that emotion dysregulation (ED) (subsuming symptoms like low frustration tolerance, irritability, ease

\footnotetext{
* Correspondence: beheshti@staff.uni-marburg.de

Department of Psychology, Child and Adolescent Clinical Psychology Group, Marburg University, Gutenbergstr.18, 35037 Marburg, Germany
}

of negative emotional experience, and emotional lability) is highly frequent in children, adolescents, and adults with ADHD ([8]; meta-analysis by [9] and qualitative reviews by $[10,11])$. About $70 \%$ of adult patients with ADHD report ED or emotional lability $[5,8,12]$. Furthermore, ED also exists in patients with ADHD not suffering from any other comorbid mental disorder [8]. Those findings reveal ED as a core component of the disorder or at least as a substantial feature in a subgroup of patients with ADHD (e.g., [4, 13]).

Two decades after Wender [14] recognized features of ED as part of the clinical presentation of adult ADHD, DSM-5 refrains from including such symptoms as

(c) The Author(s). 2020 Open Access This article is distributed under the terms of the Creative Commons Attribution 4.0 International License (http://creativecommons.org/licenses/by/4.0/), which permits unrestricted use, distribution, and 
indicative of the disorder. Instead, the DSM-5 recommends considering ED as an associated feature of ADHD supporting its diagnosis [1]. According to Kring and Sloan [15], such a limitation occurred due to the fact that ED is still a transdiagnostic concept and can be applied to psychopathological aspects of various disorders not limited to ADHD. Although focusing on emotion regulation and dysregulation might provide a) new insights into the underlying pathophysiological mechanisms (e.g., Shushakova, Ohrmann \& Pedersen, [16], b) a more accurate differentiation of symptoms and disorders (e.g., oppositional defiant disorder or conduct disorder vs. ADHS), and c) novel treatment approaches [17-19], research on ED still lacks a consensual and refined definition and depiction of ED and related constructs in general (e.g., [20, 21]), and theoretical frameworks and conceptual models of ED in ADHD in particular. Terms like ED, emotional lability, emotional instability (i.e., irregular shifting between emotional states) and emotional impulsivity (i.e., overshooting emotional responses) are often applied interchangeably or rather idiosyncratically (for a review see [17]). This lack of consensus and clarity regarding the construct of emotion regulation and ED makes summarizing and integrating empirical findings in ADHD complicated [22]. To avoid working in "conceptual and definitional chaos" ([23], p. 330), we briefly define emotion regulation, ED, and facets of ED that contribute to functional and psychosocial impairments in patients with ADHD.

Emotion regulation includes all processes that unfold over time and are related to the different emotions people have, the intensity of emotions, and how emotions are experienced and expressed [24]. The major function of ER is to shape emotional states to facilitate adaptive, goal-directed behavior in a certain situation. The most prominent model of emotion regulation is the modal model [24] that proposes five types of emotionregulation strategies [25]: (1) taking steps to influence which situation one will be exposed to (situation selection); (2) changing relevant aspects of the situation (situation modification); (3) influencing which portions of the situation are perceived and attended to (attentional deployment); (4) altering the way of thinking about it (reappraisal); and (5) directly modifying emotion-related actions (response modulation). In order to apply such emotion regulation strategies, emotions need to be recognized (i.e., perception and awareness of the self and other's verbal and nonverbal emotions) [19]. Furthermore, with respect to dysfunctional ER Ryckaert et al. [22] consider all processes that are impaired or fail to modify emotions.

Among those studies and reviews reporting on ED in ADHD, there are at present one systematic review [19] and one meta-analysis [9], both focusing on ED in childhood ADHD. The overview by Shaw et al. [19] summarizes the debate of conceptualizing ED with respect to ADHD by considering ED as a core yet distinct feature that correlates with ADHD. The metaanalysis by Graziano and Garcia [9] analyzed features of ED in children with ADHD. Distinguishing the dimensions of ED in children with ADHD, they demonstrated that such patients are more likely to experience intense emotions. The authors reported that this association between emotional reactivity and the ADHD symptom burden becomes stronger with age, a finding consistent with published reports acknowledging that ED's impairment persists over the life-span [19, 26, 27].

Relying on the ED facets derived by Graziano and Garcia [9] for children and adolescents, the goal of the present study was to conduct a meta-analysis continuing their work for adult patients with ADHD, as this has not been done so far. As previous empirical work on ADHD symptoms suggests there are differences in symptoms and their trajectories from childhood to adulthood [28-30], this might apply to ED and ED facets as well. We therefore first aimed to identify features of ED in adult ADHD with a literature review. Second, a meta-analysis was conducted to examine a) the magnitude of the associations between ADHD status (patient with ADHD vs. healthy control), ED and its facets; $b$ ) the magnitude of the associations between ADHD symptom scores, ED and its facets.

\section{Methods}

\section{Literature search}

This study has been recorded in the international prospective register of systematic reviews (Prospero) in April 2017 with the registration number CRD42017059710. A systematic literature search was undertaken using the electronic databases PubMed and PsychINFO. The literature search was consistent with the 'Preferred Reporting Items for Systematic Reviews and Meta-Analyses' (PRISMA) statement [31] and was terminated in December 2019. The Boolean expression used for our search is:

[ADHD* OR "attention deficit hyperactivity disorder" OR ADD" OR "attention deficit disorder" OR hyperkinetic"] AND ["emotion dysregulation" OR "emotion regulation" OR "mood regulation" OR "mood dysregulation" OR "affect regulation" OR "affect dysregulation" OR emotion OR labil"] AND [adult"].

\section{Inclusion and exclusion criteria}

Our search in PubMed yielded 1316 and in PsycINFO 714 abstracts. We also checked the reference lists of included studies for other studies eligible for inclusion. After removing duplicates, abstracts of all articles were screened based on pre-defined inclusion criteria independently by the first author. Inclusion criteria were: (i) 
report of any self- or third-party measure of emotion, affect, or mood (dys) regulation or emotional lability, (ii) inclusion of clinical samples of adults ( $>18$ years of age) with ADHD characterized by clinical criteria (e.g., DSM, ICD) and diagnostic procedures, (iii) inclusion of nonADHD healthy controls. Exclusion criteria were: case reports, conference abstracts, reviews, duplicates and nonEnglish studies. We included only randomized casecontrol studies that were published in peer-reviewed journals at any time from the inception of the databases. We limited our search to published studies to ensure a level of methodological adequacy and rigor among included studies and to avoid the inevitable problems with securing access to a full set of unpublished studies and the bias that would introduce [32].

After scanning a total number of 2030 studies in order to remove the duplicates, 858 studies failed to be included. In the next step, the abstracts of the studies were checked to clarify whether the subject matter is proper to our Boolean expressions. In this step, 1109 studies were excluded since the theme of ADHD in adulthood and measurements of ED were absent. In addition, studies in language other than English as well as reviews were excluded. Next, all titles fulfilling our inclusion criteria (63 studies) were reviewed in full-text. Data were collected and extracted by two independent reviewers that included ADHD status and diagnostic procedures, emotion regulation/dysregulation as defined above, gender composition (male, female), age, comorbidity, and country. When disagreement arose, reviewers consulted with each other until coming to a consensus. Ultimately, 13 studies remained for data extraction (see Fig. 1 for a flow chart of the search).

\section{Coding and data extraction}

In order to code the studies, after the final scanning, three main dimensions of ED were identified based on the narrative synthesis of the literature: Emotion recognition, emotional lability and negative emotional responses. Emotion recognition refers to the perception and awareness of the self and other's verbal and nonverbal emotions; emotional lability points to an unstable shifting between states of emotions; negative emotional responses refer to irritability and impulsivity of the

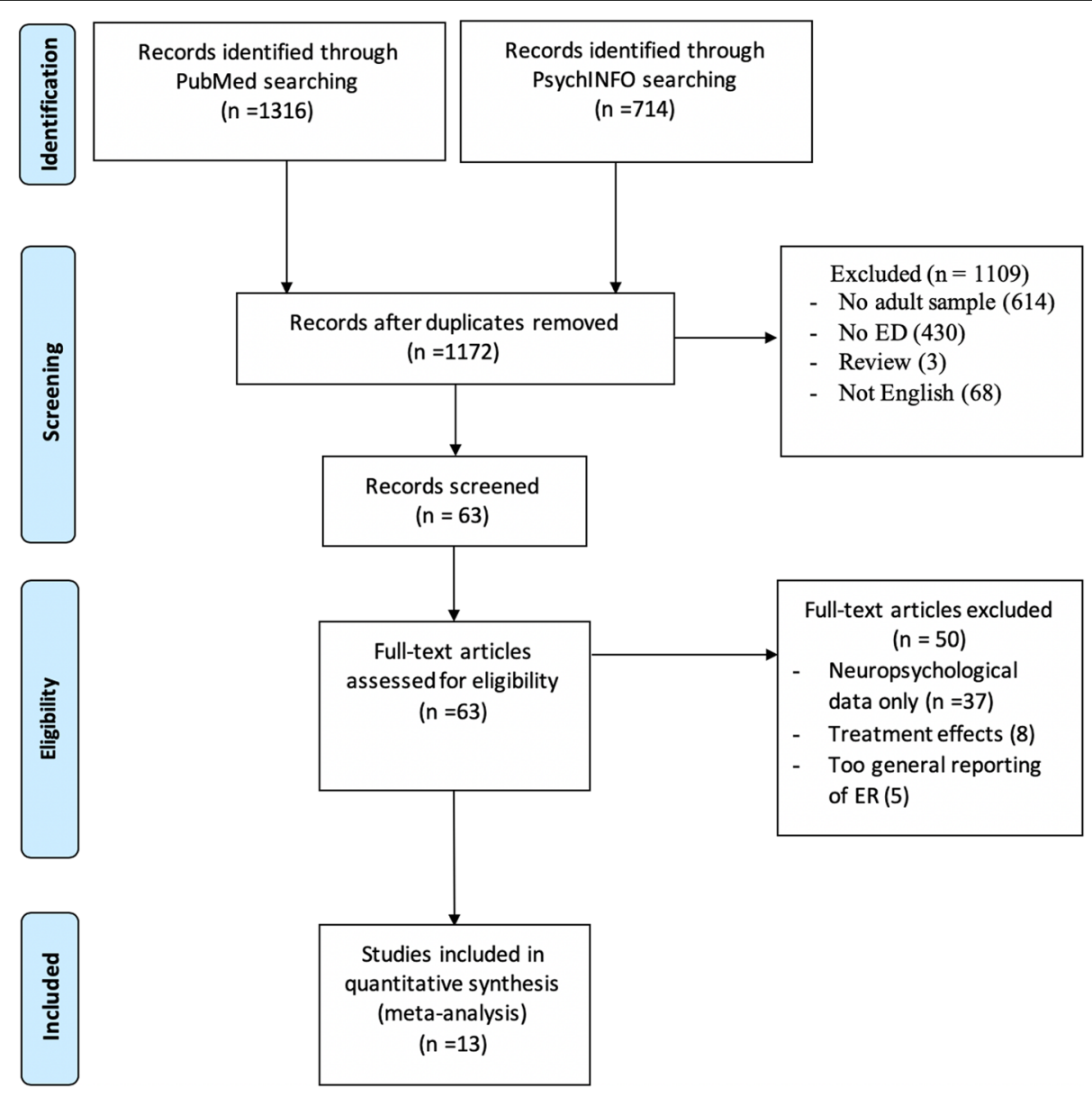

Fig. 1 Systematic Search of the Literature: PRISMA Flow-Chart 
emotional reactions [19]. For each study and in addition to information on demographics: our statistical results relied on total ED and its extracted facets (emotion recognition, emotional lability or negative emotional responses). Finally, we differentiated the included studies in two parts: the first concerned studies that examined ED between groups with and without ADHD (related to the study's question of magnitude of the associations between ADHD status, ED and its facets), and the second concerned studies that investigated ED within the groups with ADHD (related to the study's question of magnitude of the associations between ADHD symptom scores, ED and its facets).

Authors who reported ED but who had not provided enough quantitative data (e.g., only a graphic illustration) were contacted in order to request the necessary information to derive effect size estimates and confidence limits on the selected indices. When only the standard error of the mean (SEM) was reported, the standard deviation (SD) was calculated by multiplying the SEM by the square root of the sample size [33]. When descriptive statistics were reported other than the mean, SD or SEM, data were imputed by established procedures where possible [34].

\section{Effect estimation and heterogeneity}

True effect estimates were computed as adjusted standardized mean differences (Hedges' g). Meta-analysis was carried out using random-effects models and the results are reported and graphically displayed, as that better conveys data variability [33]. To estimate the average effect size, Hedges' g criteria were adopted: small = 0.2 , medium $=0.5$ and large $\geq 0.8$ [35]. Furthermore, as two studies had such small samples $(n<20)$, effect sizes were also calculated with a correction factor converting Cohen's d to Hedges' g.

Moreover, to calculate the effect sizes based on correlations, each correlation factor ( $r$ ) was converted to Fischer's z. Finally, to report all of a study's effect sizes in a corresponding unit, Fischer's $\mathrm{z}$ was converted to Hedges' $\mathrm{g}$.

To examine the consistency of results and estimate to what degree the calculated average effect sizes of a given study are representative, $\mathrm{Q}$ and $\mathrm{I}^{2}$ statistics were calculated, in which the adopted interpretation amounts are: zero or small heterogeneity for $0-40 \%$, medium heterogeneity for $40-70 \%$ and high heterogeneity for $70-90 \%$ [36].

To run all the above-mentioned analyses and demonstrate results via forest plots, we carried out initial calculations using Cochrane RevMan 5 and then repeated the calculations using Meta-Mar (1.1.0), a free online metaanalysis service developed by the first author of this study (Beheshti, in preparation).

\section{Results}

\section{Summary of systematic review}

Our systematic literature search revealed thirteen qualifying studies. We used ten of them ([8,37-47], to run our between-group analysis, as they reported their data for both groups with ADHD and healthy controls. Furthermore, four studies $[38,41,45,48]$ were included to run our within-group analysis, as they only reported data on clinical groups. Moreover, with respect to the identified dimensions of ED, Bodalski, Knouse \& Kovalev [47], Cavelti et al. [46], Corbisiero et al. [38], Irastorza [39], Reimherr et al. [48] and Surman et al. [38] reported overall measures of ED. Cavelti et al. [46] and Irastorza \& Bellon [49] additionally provided information on the specific facets of negative emotional responses and emotion recognition. The measures used by Bisch et al. [37], Miller et al. [41] and Rapport et al. [50] match the facet of emotion recognition. Mitchell et al. [41], RichardLepouriel et al. [45] and Skirrow \& Asherson [8] operationalized ED by using scales that assessed the facets of emotional lability and negative emotional responses. Rüfenacht et al., (2019) evaluated all the three dimensions of negative emotional responses, emotion recognition and emotional liability in addition to a total assessment of ED.

Moreover, and with respect to ED measurements, Cavelti et al. [46], Irastorza \& Bellon [49] and Mitchell et al. [41] adopted the long version of the Conners' Adult ADHD Rating Scale self-report that contains a subscale on Impulsivity and Emotional Lability, and used the Emotion Regulation Skills Questionnaire to measure emotion regulation skills. Furthermore, Bodalski, Knouse \& Kovalev [47] and Irastorza \& Bellon [49] employed the Deficient Emotional Self-Regulation (DESR) scale additionally, which is a section of the self-report Current Behavior Scale developed by Barkley [51] for assessing ED. Reimherr et al. [48] and Corbisiero et al. [38] assessed ED via the Affect Lability, Temper and Emotional Overreactivity subscales of the Wender-Reimherr Adult Attention Deficit Disorder Rating Scale. Miller et al. [40] and Rapport et al. [50] administered the Diagnostic Assessment of Nonverbal Accuracy (DANVA [52];) as an assessment to identify facial emotional expression. In addition, Rapport et al. [50] and Richard-Lepouriel et al. [45] administered The Affect Intensity Measure (AIM; [53] to examine experienced aspects of emotion. Richard-Lepouriel et al. [45] and Skirrow \& Asherson [8] also employed the Self-rated Affective Lability Scale (ALS [54]) to measure emotional lability and negative emotional responses. Bisch et al. [37] employed the Self-Report Emotional Intelligence Test (SREIT, [55]) to measure the ability to recognize, manage, and engage in one's own and others' emotions. Surman et al. [44] used the Deficient Emotional Self-Regulation scale (DESR [51];) to measure ED (see Table 1 for details). 
Rüfenacht et al. [43] administered The Emotion Reactivity Scale (ERS, [56]) which consists of three subscale of emotion sensitivity, intensity and persistence in order to evaluate ED.

Regarding the studies' results, Bodalski, Knouse \& Kovalev [47], Cavelti et al. [46], Corbisiero et al. [38], Irastorza [39], Reimherr et al. [48], Rüfenacht et al. [43] and Surman et al. [44] reported a significant difference between ED scores (regardless of its specific dimensions) of the groups with ADHD and healthy controls $(p<0.01, p<0.01, p<$ $0.001, p<0.01, p<0.01, p<0.001$ and $p<0.01$, respectively). Cavelti et al. [46], Irastorza \& Bellon [49] and Rüfenacht et al., [43] also reported a strong association between negative emotional responses and emotion recognition in their ADHD group ( $p<0.01$, for all of them). Moreover, the studies by Bisch et al. [37], Miller et al. [40], Rapport et al. [50] and Rüfenacht et al. [43] demonstrated a distinct difference between groups regarding the facet of emotion recognition ( $p<0.01$ for all of them). In addition, emotional lability and negative emotional responses were significantly associated in patients with ADHD in investigations by Mitchell et al. [41], RichardLepouriel et al. [45], Rüfenacht et al. [43] and Skirrow \& Asherson [8] ( $p<0.01$, for all of them).

Furthermore, the study by Corbisiero et al. [38] was the only one we included that investigated comorbidity as a moderating variable. In this context, they observed a significant difference between ADHD + ED with comorbidity and ADHD + ED with no comorbidity $(p<0.01)$. Also, the study by Cavelti et al. [45] was the only one in which ED was not only investigated in differences between patients with ADHD and healthy controls, but also it compared ED in ADHD with ED in another mental disorder: they found that patients with ADHD and borderline personality disorder exhibit significantly higher levels of emotional lability than a healthy group $(p<0.001)$. However, the difference in emotional lability was not significant between patients with ADHD and borderline personality disorders $(p=0.81)$. Table 1 provides an overview of the studies included in this meta-analysis.

\section{Summary of the meta-analysis \\ ED differences between patients with ADHD and control groups}

In answering our study's first question, namely whether groups with and without ADHD differ in emotion regulation, we noted a large average effect size of $g=1.17$ (95\% CI [0.70, 1.64], $p<0.001)$ for general emotion dysregulation according to the random effects model (for details see Fig. 2 and Table 2). In addition, with respect to specific dimensions, medium to large effect sizes were revealed for emotional lability $(g=1.20$ (95\% CI [0.57, 1.83], $p<0.001)$, negative emotional responses $(g=1.12$ (95\% CI $[0.57,1.68], p<0.001)$, and emotion recognition $(g=0.63(95 \%$ CI $[0.40,0.85], p<0.001)$. However, results of an analysis of the variance ANOVA showed that

Table 1 Summary of studies and calculated effect sizes

\begin{tabular}{|c|c|c|c|c|c|c|}
\hline Study & Age $(M \pm S D)$ & $\begin{array}{l}\text { sample } \\
\text { size }\end{array}$ & Measurements of ED & ED dimensions & design & $\begin{array}{l}\text { Effect } \\
\text { size }\end{array}$ \\
\hline [37] & $28.52 \pm 8.53$ & 54 & The Self-report of Emotional Intelligence Test & ER & Between & 0.70 \\
\hline [47] & $30.47 \pm 9.20$ & 159 & DERS & Total ED & Between & 0.92 \\
\hline [46] & $33.39 \pm 9.4$ & 135 & Impulsivity/Emotional Lability scale from the Conners' CAARS & Total ED, ER, NE & Between & 1.67 \\
\hline [38] & $32.27 \pm 10.98$ & 514 & $\begin{array}{l}\text { Emotional Dysregulation Derived from the Wender-Reimherr Adult Attention } \\
\text { Deficit Disorder } \\
\text { Rating Scale }\end{array}$ & Total ED & $\begin{array}{l}\text { Between } \\
\text { Within }\end{array}$ & $\begin{array}{l}2.04 \\
1.38\end{array}$ \\
\hline [49] & $36.29 \pm 10.71$ & 105 & Impulsivity/Emotional Lability scale from the Conners' CAARS, DERS & Total ED, ER, NE & Between & 0.85 \\
\hline [40] & $33.82 \pm 9.90$ & 51 & Diagnostic Analysis of Nonverbal Accuracy & ER & Between & 0.30 \\
\hline [41] & $23.58 \pm 5.31$ & 41 & Impulsivity/Emotional Lability scale from the Conners' CAARS & $E L, N E$ & $\begin{array}{l}\text { Between } \\
\text { Within }\end{array}$ & $\begin{array}{l}0.95 \\
2.10\end{array}$ \\
\hline [50] & $34.85 \pm 11.20$ & 56 & Affect Intensity Measure, Diagnostic Analysis of Nonverbal Accuracy & ER & Between & 0.25 \\
\hline [48] & $41.20 \pm 11.20$ & 536 & $\begin{array}{l}\text { Emotional Dysregulation Derived from the Wender-Reimherr Adult Attention } \\
\text { Deficit Disorder } \\
\text { Rating Scale }\end{array}$ & Total ED & Within & 1.09 \\
\hline [45] & $38.14 \pm 11.43$ & 198 & Affective Lability Scale, Affect Intensity Measure & $E L, N E$ & $\begin{array}{l}\text { Between } \\
\text { Within }\end{array}$ & $\begin{array}{l}0.98 \\
1.31\end{array}$ \\
\hline [43] & $35.49 \pm 12.86$ & 366 & ERS & $\begin{array}{l}\text { Total ED, EL, } \\
N E, E R\end{array}$ & Between & 0.59 \\
\hline [8] & $28.76 \pm 9.98$ & 88 & The Affective Lability Scale-Short Form & $\mathrm{EL}, \mathrm{NE}$ & Between & 1.87 \\
\hline [44] & $28.42 \pm 8.78$ & 232 & self-report Current Behavior Scale developed by R. Barkley & Total ED & Between & 2.71 \\
\hline
\end{tabular}


the difference between those specific dimensions was not significant $(\mathrm{F}=1.33$, ns).

\section{Association of ED with severity of ADHD symptoms}

Answering our study's second question regarding a correlation between ADHD symptoms in adults and emotion dysregulation dimensions, we found a strong correlation between the severity of ADHD symptoms and ED in general with an average effect size of $r=0.54$ (95\% CI [0.48, 0.61], $p<0.001$; for details see Fig. 3 and Table 3). However, our data on the correlation between the severity of ADHD symptoms and specific ED dimensions revealed that negative emotional responses contribute more with a weighted effect of $r=0.63$ (95\% CI $[0.30,0.99], p<0.001)$ whereas emotional lability revealed a slightly smaller weighted effect of $r=0.52$ (95\% CI $[0.31,0.73], p<0.001)$. However, results of an analysis of the variance (ANOVA) showed that the difference between those specific dimensions was not significant $(\mathrm{F}=$ $0.27, \mathrm{~ns})$.

\section{Heterogeneity of analysis}

$\mathrm{I}^{2}$ values are presented in Tables 2 and 3. The total heterogeneity of ED's average effect size in between-group studies was $94 \%$ and for emotion recognition, emotional lability, and negative emotional responses 40, 90 and $91 \%$, respectively. In the within-group studies, the total heterogeneity of the average effect size was $71 \%$, and for emotional lability and negative emotional responses 54 and $68 \%$, respectively.

To control for any analysis bias, we used funnel plots and Fail-safe $\mathrm{N}$ tests. Our between-group analysis results showed that the funnel plot is asymmetric, with the smaller studies tending toward the left of the average effect size. This may indicate that there are studies missing from the right side. Consequently, were there no such probable bias, the average effect size could be larger than the aforementioned amount. In addition, our Fail-safe N test results showed that 1481 studies need to be added
Table 2 Effect Sizes for differences in ED dimensions between adults with and without ADHD

\begin{tabular}{|c|c|c|c|c|}
\hline & ED & ER & EL & $\mathrm{NE}$ \\
\hline Hedges'g & 1.17 & 0.63 & 1.20 & 1.12 \\
\hline $95 \% \mathrm{Cl}$ & {$[0.70,1.64]$} & {$[0.40,0.85]$} & {$[0.57,1.83]$} & {$[0.57,1.68]$} \\
\hline Hedges'g Criteria & Large & Moderate & Large & Large \\
\hline $1^{2}$ & $94 \%$ & $40 \%$ & $90 \%$ & $91 \%$ \\
\hline Criteria of $\mathrm{I}^{2}$ & High & Moderate & High & High \\
\hline Number of studies & 12 & 6 & 4 & 6 \\
\hline Number of participants & 1926 & 767 & 695 & 933 \\
\hline
\end{tabular}

ED Emotion Dysregulation, ER Emotion Recognition, EL Emotional Lability, NE Negative Emotions. $I^{2}$ : Heterogeneity of the study

to our analysis to reduce the effect size to statistical non-significance (for details see Fig. 4a).

Concerning our within-group analysis, we noted an asymmetric funnel plot with the smaller studies leaning to the right of the average effect size. Four studies were included at this level with sample sizes of 539, 396, 150 and 18 ([38, 41, 45, 48]), respectively. This may indicate studies missing from the left side. Therefore, the average effect size might be smaller than the current estimate. In addition, our Fail-safe $\mathrm{N}$ test results showed that 732 studies need to be added to our analysis to reduce the effect size to statistical non-significance (for details see Fig. 4b).

\section{Discussion}

The present meta-analysis was conducted to establish aspects of ED in adulthood ADHD and to differentiate such aspects between ADHD and healthy control groups. Another goal of our study was to assess any association between ED's features and ADHD symptoms. In line with these research objectives, we identified dimensions of ED based on our adopted conceptual models of ED (i.e., emotion regulation model by Gross [57], and regarding features of ED in adulthood ADHD that focused on the studies we selected for this study. Three dimensions of

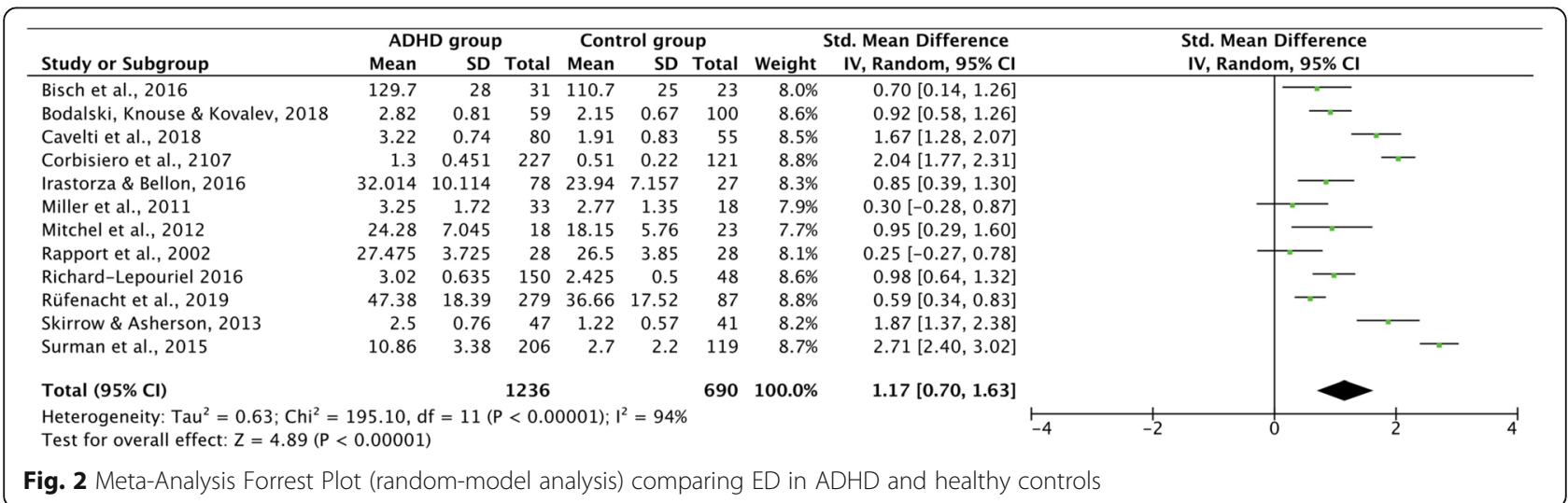




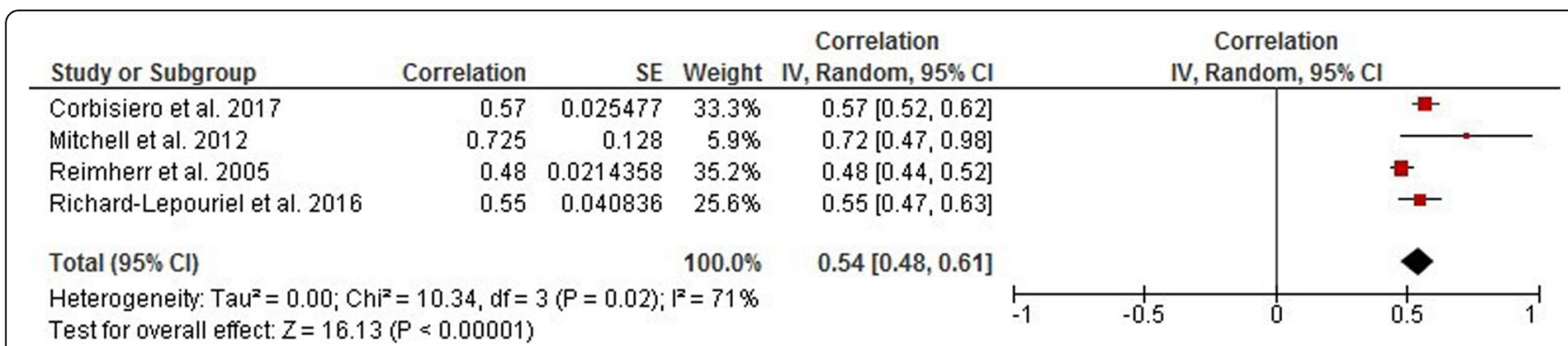

Fig. 3 Meta-Analysis Forrest Plot on Correlation Coefficients between measures of ADHD symptom severity and ED

emotion recognition (ER), emotional lability (EL), and negative emotional responses (NE) were distinguished.

We then categorized the 13 studies selected $(N=2535)$ by two labels of between-group studies (10), in which data was reported on both groups with ADHD and health controls, and within-group studies (4), in which only data on patients with ADHD was available. At the between-group analysis level, we found that compared to a control group, emotion dysregulation is significantly more pronounced in adults with ADHD with a large effect size (Hedges' $g=1.17$ ). Furthermore, regarding ED's intermediate dimensions, emotional lability revealed the largest effect size (Hedges' $g=1.20$ ). Previous studies demonstrated the relevance of ED for mental and somatic health in general (e.g., $[58,59])$ and for ADHS in particular [19]. Barkley \& Fischer [60] demonstrated that adult patients with persisting ADHD reported worse ED than healthy control participants. In another example, Corbisiero et al. [7] differentiated affective lability from reactivity and temper as core features of ED; they reported higher rates of ED in adults with ADHD. Compatible with these findings, our results support the significant difference between the rates of ED in adulthood ADHD and control groups. Skirrow \& Asherson [8] also reported that emotional lability contributed independently to impairing the daily life of adults with ADHD.

Table 3 Effect sizes for differences in ED dimensions in adults with ADHD

\begin{tabular}{|c|c|c|c|}
\hline & ED & EL & $\mathrm{NE}$ \\
\hline Correlation Coefficient (r) & 0.54 & 0.52 & 0.63 \\
\hline 95\% Cl (random-model) & {$[0.48,0.61]$} & {$[0.31,0.73]$} & {$[0.30,0.99]$} \\
\hline r criteria & Large & Large & Large \\
\hline $1^{2}$ & $71 \%$ & $54 \%$ & $68 \%$ \\
\hline Criteria of $\mathrm{I}^{2}$ & High & Medium & Medium \\
\hline Number of studies & 4 & 2 & 2 \\
\hline Number of samples & 1097 & 168 & 168 \\
\hline
\end{tabular}

ED Emotion Dysregulation, ER Emotion Recognition, EL Emotional Lability, NE Negative Emotions. $I^{2}$ : Heterogeneity of the study
Finally, we observed a strong correlation between the severity of ADHD symptoms and ED $(r=0.54)$. In terms of ED, dimensions, negative emotional responses exhibited the strongest correlation with the core ADHD symptoms $(r=0.63)$ - findings that concur with the literature $[16,26,61]$, and that are also compatible with the study by Graziano \& Garcia [9] that reported a stronger correlation between emotional responses and ADHD symptoms in older adolescents.

Our results demonstrate that emotional lability plays both a significant role in differentiating clinical groups with ADHD from healthy controls and a strong correlation between negative emotional responses and ADHD symptom severity. Referring to adopted conceptual models, these findings might be explained by considering the following: First, the literature suggests that concerning ADHD's epidemiology in adults, the evidence that hyperactive-impulsive symptoms seem to remit in older age groups may be attributable to adaptive strategies patients develop over the life-span, while inattention symptoms seem to persist [62-64];). In this regard, these symptoms might be correlated better with impaired situation identification that requires attention processes, as well as a lack of strategies for monitoring emotion regulation processes - which in turn would trigger higher rates of emotional lability in adults with ADHD. Second, the severity of ADHD symptoms in adults correlated significantly with negative externalizing behaviors such as aggression and irritation, as articulated by Posner et al. [65] in their dyscontrol hypothesis and affectivity hypothesis. Based on dyscontrol hypothesis, impairments in the capacity of inhibiting the emotional responses occurs significantly more in hyperactive subgroups of ADHD. In this regard, functional neuroimaging shows anomalies within frontolimbic circuits. According to the affectivity hypothesis, negativeemotionally-responsive behavior in ADHD patients emerges through the route of dysfunctional emotional processing associated with the amygdala and medial prefrontal cortex [65]. Emotion recognition seems to be a more serious problem in young people with ADHD. As the Graziano \& Garcia [9] analysis implied, emotion 


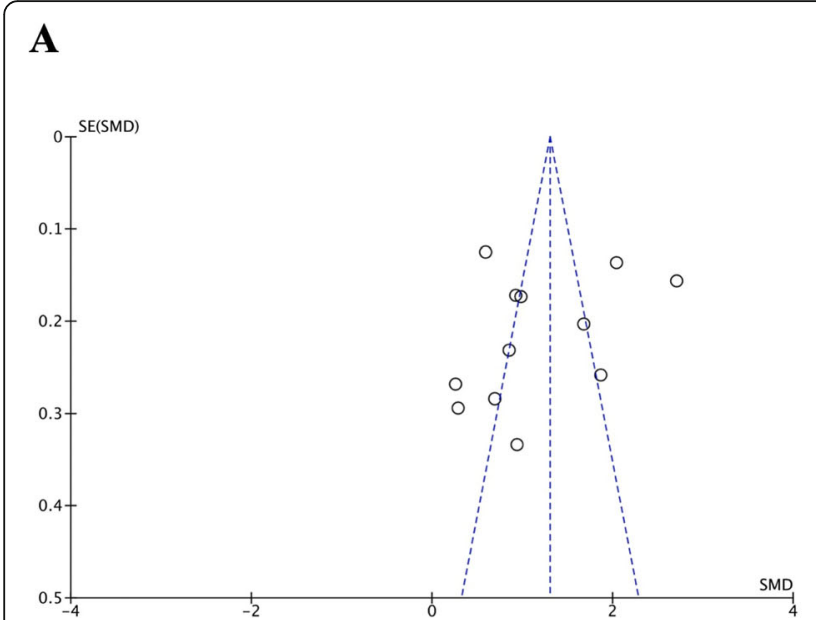

\section{B}

Fig. 4 Funnel Plot a) refers to the meta-analysis comparing ED in ADHD and healthy controls $\mathbf{b}$ ) refers to the meta-analysis of the correlation coefficients between measures of ADHD symptom severity and ED

recognition skills are weaker in younger children, and as cognitive abilities develop and adapt, emotion recognition improves. Therefore, although emotion recognition remains a feature of ED in adulthood ADHD, emotional lability and negative emotional responses play a more pronounced role in the ED-associated psychopathology of adults with ADHD.

\section{Practical implications of the results}

Most likely, ED in adults with ADHD is a problem persisting from childhood that either was addressed with no specific intervention (as juvenile ADHD treatment is predominantly pharmacological [66, 67];)), or that was therapy-resistant and continues to be a obvious feature over the course of the disorder $[68,69]$. In this regard, our study findings support the consideration of therapeutic approaches entailing ED improvement strategies and reinforcing emotion regulation skills, in addition to standard interventions for the disorder [4, 70]. Furthermore, as our results demonstrate, such therapeutic strategies and interventions would be advisable to focus on a general ED impairment in adults with ADHD while considering emotional lability and negative emotional responses as aspects of ED that need to be targeted in adults with ADHD. Moreover, there is strong evidence of the effectiveness of pharmacological [27, 71, 72];) and psychotherapeutic interventions in alleviating emotion dysregulation and the disorder's core symptoms $[4,6$, $11,73,74]$. In light of the problem of diagnosing ADHD in adulthood and the lack of specific criteria for adults, our meta-analysis findings suggest that adopting an approach that addresses aspects of ED in the diagnosis and

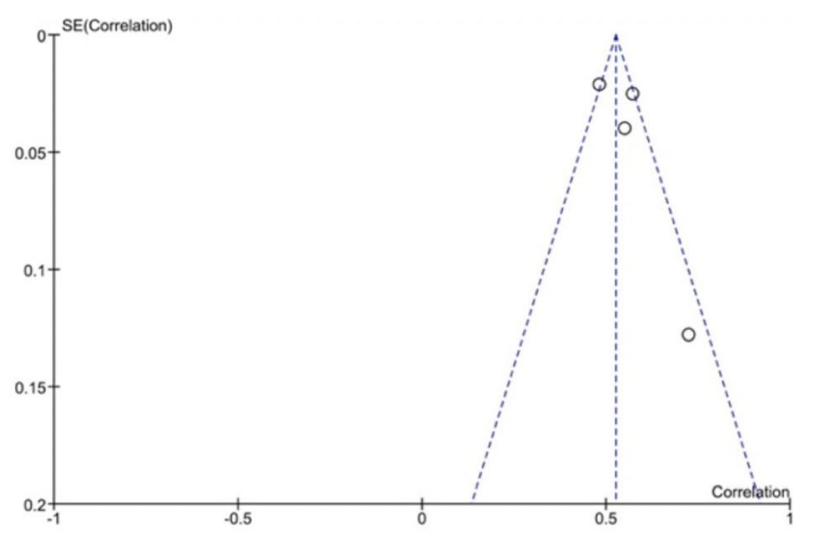

treatment of adults with ADHD would yield a valuable supplementary benefit.

\section{Study limitations}

We assumed that different measures of ED are contingent, though that might not always be the case. As the adopted conceptual models of ED converged in the studies included in our meta-analysis, we did not analyze them accordingly.

Moreover, reviews have shown that moderators such as gender and cognitive functions ([75-77]; as well as the presence of comorbidity [3] or medication [6] play a significant role in ADHD. Therefore, if emotion dysregulation is assumed to be a main feature of ADHD in adults, controlling for such moderators should be part of a meta-analysis. However, as the studies included contained a paucity of such statistical data, we could not perform meta-regressions that might have shed light on such moderators.

Finally, our funnel plots and Fail-safe $\mathrm{N}$ test results imply that (probably) missing studies and t hus omitted from our meta-analysis contributed to asymmetrically distributed effect sizes. In between-group analysis, missing studies would strengthen, and in within-group analysis weaken effects. In addition, the lesser degree of heterogeneity $(71 \%)$ in our within-group analysis compared to the between-group analysis might be a samplesize problem.

\section{Conclusions}

In conclusion, our results from the present meta-analysis focusing on the role of emotion dysregulation in adulthood ADHD imply that compared to a control group, ED 
is a distinct feature of adult ADHD. Furthermore, the severity of ADHD symptoms significantly correlates with dimensions of ED such as emotional lability, emotion recognition, and emotional responses, replicating other studies in the field (e. g., [78]). In addition, classic domains of inattention, hyperactivity, and impulsivity do not sufficiently explain the entire symptom spectrum. In this respect, assessing and targeting emotion regulation in clinical practice might prove to be a valuable strategy for diagnosing and treating adult ADHD. Moreover, future research should clarify how ED interacts with adult ADHD symptoms, comorbid conditions, and other moderators such as demographics.

\section{Abbreviations \\ ADHD: Attention deficit/hyperactivity disorder; AIM: Affect Intensity Measure; ALS: Affective Lability Scale; DANVA: Diagnostic Assessment of Nonverbal Accuracy; DESR: Deficient Emotional Self-Regulation; ED: Emotion dysregulation; EL: Emotional lability; ER: Emotion recognition; ERS: Emotion Reactivity Scale; NE: Negative emotional responses; SD: Standard deviation; SEM: Standard error of the mean; SREIT: Self-Report Emotional Intelligence Test}

\section{Acknowledgements}

Not applicable.

\section{Authors' contributions}

$A B$ designed the meta-analysis, and conducted the literature search, data coding, and statistical analysis. MLC did the studies' reliability coding. HC provided expert advice throughout these processes. $A B$ wrote the first draft of the manuscript and all authors contributed to and have approved the final manuscript.

\section{Funding}

No specific grant was received funding agencies in the public, commercial, or not-for-profit sectors to conduct this research. .

\section{Availability of data and materials}

The datasets used and analyzed during the present study are available from the corresponding author on reasonable request.

\section{Ethics approval and consent to participate}

Not applicable.

\section{Consent for publication}

Not applicable.

\section{Competing interests}

The authors declare that they have no competing interests.

Received: 21 September 2018 Accepted: 13 January 2020 Published online: 12 March 2020

\section{References}

1. Association AP. Diagnostic and statistical manual of mental disorders (DSM$\left.5^{\oplus}\right)$ : American psychiatric pub; 2013.

2. Barkley RA. Differential diagnosis of adults with ADHD: the role of executive function and self-regulation. J Clin Psychiatry. 2010;71:e17.

3. Kessler RC, Adler L, Barkley R, Biederman J, Conners CK, Demler O, et al. The prevalence and correlates of adult ADHD in the United States: results from the National Comorbidity Survey Replication. Am J Psychiatry. 2006;163:71623. https://doi.org/10.1176/ajp.2006.163.4.716.

4. Retz W, Stieglitz R-D, Corbisiero S, Retz-Junginger P, Rösler M. Emotional dysregulation in adult ADHD: what is the empirical evidence? Expert Rev Neurother. 2012;12:1241-51. https://doi.org/10.1586/ern.12.109.

5. Barkley RA, Murphy KR, Fischer M. Adult ADHD: What the science says. New York: Guilford; 2008.
6. Bunford N, Dawson AE, Evans SW, Ray AR, Langberg JM, Owens JS, et al. The Difficulties in Emotion Regulation Scale-Parent Report: A Psychometric Investigation Examining Adolescents With and Without ADHD. Assessment. 2018:1073191118792307.

7. Corbisiero S, Stieglitz R-D, Retz W, Rösler M. Is emotional dysregulation part of the psychopathology of ADHD in adults? ADHD Atten Defic Hyperact Disord. 2013;5:83-92.

8. Skirrow C, Asherson P. Emotional lability, comorbidity and impairment in adults with attention-deficit hyperactivity disorder. J Affect Disord. 2013;147: 80-6. https://doi.org/10.1016/j.jad.2012.10.011.

9. Graziano PA, Garcia A. Attention-deficit hyperactivity disorder and children's emotion dysregulation: a meta-analysis. Clin Psychol Rev. 2016;46:106-23. https://doi.org/10.1016/j.cpr.2016.04.011.

10. Martel MM. Research review: a new perspective on attention-deficit/ hyperactivity disorder: emotion dysregulation and trait models. J Child Psychol Psychiatry. 2009;50:1042-51. https://doi.org/10.1111/j.1469-7610. 2009.02105.X.

11. Ramsay JR, Rostain AL. Adult ADHD research: current status and future directions. J Atten Disord. 2008:11:624-7. https://doi.org/10.1177/ 1087054708314590.

12. Skirrow C, Ebner-Priemer U, Reinhard I, Malliaris Y, Kuntsi J, Asherson P. Everyday emotional experience of adults with attention deficit hyperactivity disorder: evidence for reactive and endogenous emotional lability. Psychol Med. 2014;44:3571-83. https://doi.org/10.1017/S0033291714001032.

13. Hirsch O, Chavanon M, Riechmann E, Christiansen H. Emotional dysregulation is a primary symptom in adult attention-deficit/hyperactivity disorder (ADHD). J Affect Disord. 2018;232:41-7. https://doi.org/10.1016/j.jad. 2018.02.007.

14. Wender PH. Attention-deficit hyperactivity disorder in adults. Psychiatr Clin N Am. 1995;21(4):761-74

15. Kring AM, Sloan DM. Emotion regulation and psychopathology: A transdiagnostic approach to etiology and treatment: Guilford Press; 2009.

16. Shushakova A, Ohrmann P, Pedersen A. Exploring deficient emotion regulation in adult ADHD: electrophysiological evidence. Eur Arch Psychiatry Clin Neurosci. 2018;268:359-71. https://doi.org/10.1007/s00406-017-0826-6.

17. Faraone SV, Rostain AL, Blader J, Busch B, Childress AC, Connor DF, Newcorn JH. Practitioner review: emotional dysregulation in attentiondeficit/hyperactivity disorder - implications for clinical recognition and intervention. J Child Psychol Psychiatry. 2018. https://doi.org/10.1111/jcpp. 12899.

18. Hoxhaj E, Sadohara C, Borel P, D'Amelio R, Sobanski E, Müller H, et al. Mindfulness vs psychoeducation in adult ADHD: a randomized controlled trial. Eur Arch Psychiatry Clin Neurosci. 2018;268:321-35. https://doi.org/10. 1007/s00406-018-0868-4.

19. Shaw P, Stringaris A, Nigg J, Leibenluft E. Emotion dysregulation in attention deficit hyperactivity disorder. Am J Psychiatry. 2014;171:276-93. https://doi. org/10.1176/appi.ajp.2013.13070966.

20. Cole PM, Martin SE, Dennis TA. Emotion regulation as a scientific construct: methodological challenges and directions for child development research. Child Dev. 2004;75:317-33.

21. Izard CE. Emotion theory and research: highlights, unanswered questions, and emerging issues. Annu Rev Psychol. 2009;60:1-25. https://doi.org/10. 1146/annurev.psych.60.110707.163539.

22. Ryckaert C, Kuntsi J, Asherson P. Emotional dysregulation and ADHD. In: Oxford Textbook of Attention Deficit Hyperactivity Disorder (Oxford Library of Psychology), vol. 103; 2018.

23. Buck R. Mood and emotion: a comparison of five contemporary views. Psychol Inq. 1990;1:330-6.

24. Gross JJ, Thompson R. Emotion Regulation: Conceptual Foundations. Handbook of Emotion Regulation; 2007.

25. Werner K, Gross JJ. Emotion regulation and psychopathology: A conceptual framework. In: Kring AM, Sloan DM, editors. Emotion regulation and psychopathology: A transdiagnostic approach to etiology and treatment: The Guilford Press; 2009. p. 13-37.

26. Helfer B, Cooper RE, Bozhilova N, Maltezos S, Kuntsi J, Asherson P. The effects of emotional lability, mind wandering and sleep quality on ADHD symptom severity in adults with ADHD. European Psychiatry. 2019;55:45-51.

27. Schweren L, Hoekstra P, van Lieshout M, Oosterlaan J, Lambregts-Rommelse $\mathrm{N}$, Buitelaar J, et al. Long-term effects of stimulant treatment on ADHD symptoms, social-emotional functioning, and cognition. Psychol Med. 2019; 49(2):217-23. 
28. Asherson P. ADHD across the lifespan. Medicine. 2016;44:683-6.

29. Fayyad J, Sampson NA, Hwang I, Adamowski T, Aguilar-Gaxiola S, AlHamzawi A, et al. The descriptive epidemiology of DSM-IV adult ADHD in the World Health Organization world mental health surveys. Atten Defic Hyperact Disord. 2017;9:47-65. https://doi.org/10.1007/s12402-016-0208-3.

30. Thomas R, Sanders S, Doust J, Beller E, Glasziou P. Prevalence of attentiondeficit/hyperactivity disorder: a systematic review and meta-analysis. Pediatrics. 2015;135:e994-1001. https://doi.org/10.1542/peds.2014-3482.

31. Moher D. Preferred reporting items for systematic reviews and metaanalyses: the PRISMA statement. Ann Intern Med. 2009;151:264. https://doi. org/10.7326/0003-4819-151-4-200908180-00135.

32. Sonuga-Barke EJ, Brandeis D, Cortese S, Daley D, Ferrin M, Holtmann M, et al. Nonpharmacological interventions for ADHD: systematic review and meta-analyses of randomized controlled trials of dietary and psychological treatments. Am J Psychiatr. 2013:170(3):275-89.

33. Higgins JPT, Green S. Cochrane handbook for systematic reviews of interventions. Oxford: Cochrane Collaboration; 2011.

34. Glass GV, McGaw B, Smith ML. Meta-Analysis in Social Research. Beverly Hills: Sage Publications; 1981.

35. Turner HM, Bernard RM. Calculating and synthesizing effect sizes. Contemp Issues Commun Sci Disord. 2006;33:42-55.

36. Higgins JP, Thompson SG, Deeks JJ, Altman DG. Measuring inconsistency in meta-analyses. BMJ. 2003;327(7414):557-60. https://doi.org/10.1136/bmj.327. 7414.557.

37. Bisch J, Kreifelts B, Bretscher J, Wildgruber D, Fallgatter A, Ethofer T. Emotion perception in adult attention-deficit hyperactivity disorder. J Neural Transm. 2016;123:961-70

38. Corbisiero S, Mörstedt B, Bitto H, Stieglitz R-D. Emotional Dysregulation in adults with attention-deficit/hyperactivity disorder-validity, predictability, severity, and comorbidity. J Clin Psychol. 2017;73:99-112.

39. Irastorza LJ. Questions about adult ADHD patients: dimensional diagnosis, emotion Dysregulation, Competences and Empathy. Acta Psychopathol. 2016. https://doi.org/10.4172/2469-6676.100069.

40. Miller M, Hanford RB, Fassbender C, Duke M, Schweitzer JB. Affect recognition in adults with ADHD. J Atten Disord. 2011;15:452-60. https:// doi.org/10.1177/1087054710368636.

41. Mitchell JT, Robertson CD, Anastopolous AD, Nelson-Gray RO, Kollins SH. Emotion Dysregulation and emotional impulsivity among adults with attention-deficit/hyperactivity disorder: results of a preliminary study. J Psychopathol Behav Assess. 2012;34:510-9. https://doi.org/10.1007/s10862012-9297-2.

42. Rapport LJ, Friedman SR, Tzelepis A, van Voorhis A. "Experienced emotion and affect recognition in adult attention-deficit hyperactivity disorder": Correction to Rapport et al. (2002). Neuropsychology. 2002;16:369. https:// doi.org/10.1037/0894-4105.16.3.369.

43. Rüfenacht E, Euler S, Prada P, Nicastro R, Dieben K, Hasler R, et al. Emotion dysregulation in adults suffering from attention deficit hyperactivity disorder (ADHD), a comparison with borderline personality disorder (BPD). Borderline Personal Disord Emot Dysregul. 2019;6(1). https://doi.org/10.1186/s40479019-0108-1.

44. Surman CBH, Biederman J, Spencer T, Miller CA, Petty CR, Faraone SV. Neuropsychological deficits are not predictive of deficient emotional selfregulation in adults with ADHD. J Atten Disord. 2015;19:1046-53. https://doi. org/10.1177/1087054713476548.

45. Richard-Lepouriel H, Etain B, Hasler R, Bellivier F, Gard S, Kahn J-P, et al. Similarities between emotional dysregulation in adults suffering from ADHD and bipolar patients. J Affect Disord. 2016;198:230-6. https://doi.org/10. 1016/j.jad.2016.03.047.

46. Cavelti M, Corbisiero S, Bitto H, Moerstedt B, Newark P, Faschina S, et al. A comparison of self-reported emotional regulation skills in adults with attention-deficit/hyperactivity disorder and borderline personality disorder. J Atten Disord. 2017;23(12):1087054717698814.

47. Bodalski EA, Knouse LE, \& Kovalev D. Adult ADHD, Emotion Dysregulation, and Functional Outcomes: Examining the Role of Emotion Regulation Strategies. J Psychopathol Behav Assess, 2018;41(1):81-92.

48. Reimherr FW, Marchant BK, Strong RE, Hedges DW, Adler L, Spencer TJ, et al. Emotional dysregulation in adult ADHD and response to atomoxetine. Biol Psychiatry. 2005;58:125-31. https://doi.org/10.1016/j.biopsych.2005.04. 040.

49. Irastorza LJ, Bellon JM. Adult ADHD Patients: Emotional Dysregulation and Personality Dimensions. Age. 2016;34:10-5.
50. Rapport L, Friedman SL, Tzelepis A, Van Voorhis A. Experienced emotion and affect recognition in adult attention-deficit hyperactivity disorder. Neuropsychology. 2002;16(1):102.

51. Barkley RA. Behavioral inhibition, sustained attention, and executive functions: constructing a unifying theory of ADHD. Psychol Bull. 1997;121:65.

52. Nowicki S, Duke MP. Individual differences in the nonverbal communication of affect: the diagnostic analysis of nonverbal accuracy scale. J Nonverbal Behav. 1994;18(1):9-35.

53. Larsen RJ, Diener E. A multitrait-multimethod examination of affect structure: Hedonic level and emotional intensity. Pers Individ Diff. 1985;6(5): 631-6.

54. Oliver MN, Simons JS. The affective lability scales: Development of a shortform measure. Pers Individ Diff. 2004:37(6):1279-88.

55. Schutte NS, Malouff JM, Hall LE, Haggerty DJ, Cooper JT, Golden CJ, Dornheim L. Development and validation of a measure of emotional intelligence. Personal Individ Differ. 1998;25(2):167-77.

56. Nock MK, Wedig MM, Holmberg EB, Hooley JM. The emotion reactivity scale: development, evaluation, and relation to self-injurious thoughts and behaviors. Behav Ther. 2008;39(2):107-16.

57. Gross JJ. Emotion regulation: taking stock and moving forward. Emotion. 2013;13(3):359.

58. Kring AM. Handbook of emotions: emotion disturbances as transdiagnostic processes in psychopathology. 2nd ed. New York: Guilford Press; 2008.

59. Kring AM. The future of emotion research in the study of psychopathology. Emot Rev. 2010;2:225-8. https://doi.org/10.1177/1754073910361986.

60. Barkley RA, Murphy KR, Fischer M. ADHD in adults: What the science says: Guilford Press; 2010

61. Evren B, Evren C, Dalbudak E, Topcu M, Kutlu N. Relationship of internet addiction severity with probable ADHD and difficulties in emotion regulation among young adults. Psychiatry Res. 2018;269:494-500.

62. Polanczyk GV, Willcutt EG, Salum GA, Kieling C, Rohde LA. ADHD prevalence estimates across three decades: an updated systematic review and metaregression analysis. Int J Epidemiol. 2014;43:434-42. https://doi.org/10.1093/ ije/dyt261.

63. Simon V, Czobor P, Bálint S, Mészáros A, Bitter I. Prevalence and correlates of adult attention-deficit hyperactivity disorder: meta-analysis. $\mathrm{Br}$ Psychiatry. 2009;194:204-11. https://doi.org/10.1192/bjp.bp.107.048827.

64. Barkley RA. Distinguishing sluggish cognitive tempo from ADHD in children and adolescents: executive functioning, impairment, and comorbidity. J Clin Child Adolesc Psychol. 2013:42(2):161-73.

65. Posner J, Kass E, Hulvershorn L. Using stimulants to treat ADHD-related emotional lability. Curr Psychiatry Rep. 2014;16(10):478.

66. Rowles BM, Findling RL. Review of pharmacotherapy options for the treatment of attention-deficit/hyperactivity disorder (ADHD) and ADHD-like symptoms in children and adolescents with developmental disorders. Dev Disabil Res Rev. 2010;16:273-82. https://doi.org/10.1002/ddrr.120.

67. Daughton JM, Kratochvil CJ. Review of ADHD pharmacotherapies: advantages, disadvantages, and clinical pearls. J Am Acad Child Adolesc Psychiatry. 2009;48:240-8. https://doi.org/10.1097/CHI.0b013e318197748f.

68. Peyre $H$, Speranza M, Cortese S, Wohl M, Purper-Ouakil D. Do ADHD children with and without child behavior checklist-dysregulation profile have different clinical characteristics, cognitive features, and treatment outcomes? J Atten Disord. 2015;19(1):63-71.

69. Rösler M, Retz W, Fischer R, Ose C, Alm B, Deckert J, et al. Twenty-four-week treatment with extended release methylphenidate improves emotional symptoms in adult ADHD. World J Biol Psychiatry. 2010;11(5):709-18.

70. Cole P, Weibel S, Nicastro R, Hasler R, Dayer A, Aubry JM, et al. CBT/DBT skills training for adults with attention deficit hyperactivity disorder (ADHD). Psychiatr Danub. 2016;28(Suppl 1):103-7.

71. Lenzi F, Cortese S, Harris J, Masi G. Pharmacotherapy of emotional dysregulation in adults with ADHD: a systematic review and meta-analysis. Neurosci Biobehav Rev. 2018;84:359-67. https://doi.org/10.1016/j.neubiorev. 2018.08.010.

72. Moukhtarian TR, Cooper RE, Vassos E, Moran P, Asherson P. Effects of stimulants and atomoxetine on emotional lability in adults: a systematic review and meta-analysis. Eur Psychiatry. 2017;44:198-207. https://doi.org/ 10.1016/j.eurpsy.2017.05.021

73. Hesslinger B, van Tebartz EL, Nyberg E, Dykierek P, Richter H, Berner M, Ebert D. Psychotherapy of attention deficit hyperactivity disorder in adults-a pilot study using a structured skills training program. Eur Arch Psychiatry Clin Neurosci. 2002;252:177-84. https://doi.org/10.1007/s00406-002-0379-0. 
74. Philipsen A, Graf E, van Tebartz EL, Jans T, Warnke A, Hesslinger B, et al.

Evaluation of the efficacy and effectiveness of a structured disorder tailored psychotherapy in ADHD in adults: study protocol of a randomized

controlled multicentre trial. ADHD Atten Defic Hyperact Disord. 2010;2:20312. https://doi.org/10.1007/s12402-010-0046-7.

75. Boonstra AM, Oosterlaan J, Sergeant JA, Buitelaar JK. Executive functioning in adult ADHD: a meta-analytic review. Psychol Med. 2005;35:1097-108.

76. Bridgett DJ, Walker ME. Intellectual functioning in adults with ADHD: a meta-analytic examination of full scale IQ differences between adults with and without ADHD. Psychol Assess. 2006;18:1.

77. Gershon J. A meta-analytic review of gender differences in ADHD. J Atten Disord. 2002;5:143-54. https://doi.org/10.1177/108705470200500302.

78. Hirsch O, Chavanon M, Christiansen H. Emotional dysregulation subgroups in patients with adult attention-deficit/hyperactivity disorder (ADHD): a cluster analytic approach. Sci Rep. 2019. https://doi.org/10.1038/s41598-01942018-y.

\section{Publisher's Note}

Springer Nature remains neutral with regard to jurisdictional claims in published maps and institutional affiliations.

Ready to submit your research? Choose BMC and benefit from:

- fast, convenient online submission

- thorough peer review by experienced researchers in your field

- rapid publication on acceptance

- support for research data, including large and complex data types

- gold Open Access which fosters wider collaboration and increased citations

- maximum visibility for your research: over $100 \mathrm{M}$ website views per year

At $B M C$, research is always in progress.

Learn more biomedcentral.com/submissions 